\title{
Unusual Foreign Bodies of Surgical Discovery on a Traumatic Spine
}

\section{Habib Abdoul Karim Ouiminga ${ }^{{ }^{*}}$, Julien T. Savadogo², Denléwendé Sylvain Zabsonré3, Anatole Jean Innocent Ouedraogo', Diane Ndzana ${ }^{2}$, Mengyou Li1 ${ }^{1}$, Désiré Harouna Sankara ${ }^{4}$, Magatte Gaye ${ }^{5}$}

\author{
${ }^{1}$ Department of Orthopedics Traumatology and Neurosurgery, CHU de Tingandogo, Ouagadougou, Burkina Faso \\ ${ }^{2}$ Department of Thoracic and Vascular Surgery, CHU de Tingandogo, Ouagadougou, Burkina Faso \\ ${ }^{3}$ Department of Neurosurgery, CHU Yalgado Ouedraogo, Ouagadougou, Burkina Faso \\ ${ }^{4}$ Department of Imagery, Ouagadougou Medical Imaging Center, Ouagadougou, Burkina Faso \\ ${ }^{5}$ Department of Neurosurgery, Hôpital General Grand Yoff, Dakar, Senegal \\ Email: ^ouimkar@gmail.com
}

How to cite this paper: Ouiminga, H.A.K., Savadogo, J.T., Zabsonré, D.S., Ouedraogo, A.J.I., Ndzana, D., Li, M., Sankara, D.H. and Gaye, M. (2020) Unusual Foreign Bodies of Surgical Discovery on a Traumatic Spine. World Journal of Neuroscience, 10, $15-21$.

https://doi.org/10.4236/wjns.2020.101003

Received: November 24, 2019

Accepted: December 24, 2019

Published: December 27, 2019

Copyright $\odot 2020$ by author(s) and Scientific Research Publishing Inc. This work is licensed under the Creative Commons Attribution International License (CC BY 4.0).

http://creativecommons.org/licenses/by/4.0/

\begin{abstract}
Introduction: Para-spinal non-metallic foreign bodies (fabrics or plastics) are rare and poorly documented. They are often unknown and discovered at the stage of infectious complications and present big therapeutic challenges. We report a rare case of three para-spinal foreign bodies (fabric, plastic and postoperative gauze) discovered during surgery of a traumatic thoracic spine. Case report: A 32-year-old man admitted for a polytrauma (collision motorcycle-cart). The admission examination noted closed trauma of the thoracic spine, an ASIA score of A, dyspnea, a penetrating wound of the left side of the chest. The CT scan showed a compressive left pleural effusion, multiple ribs fractures, pulmonary contusion, unstable fracture of fifth and sixth thoracic vertebrae associated with posterior epidural hematoma responsible for medullar compression. There was a rounded, para-spinal image, dotted with small areas of low density, air bubbles. We lifted the vital emergency by draining the left pleural effusion, debriding the penetrating chest wound, and administering broad-spectrum antibiotic therapy. Fourteen days later, we decided to stabilize the spine. After a posterior approach, we discovered free pus and para-vertebral three foreign bodies. Enterobacter spp. was isolated in pus susceptible to imipenem. The immediate operative follow-up was simple. Conclusion: The best treatment remains preventive by simple measures, exploration of penetrating wounds, repeated count and careful verification of gauze, because the infectious complications that they generate are source of mortality and serious medico-legal implications.
\end{abstract}




\section{Keywords}

Textiloma, Foreign Body, Spine, Traumatism, Surgery

\section{Introduction}

Para or intra-spinal metallic foreign bodies are often encountered mainly in ballistic traumas. Para-spinal foreign bodies, however, are rare and poorly documented in published journals [1] [2]. The rare cases described were observed after abdominal or thoracic surgery [2] [3] [4]. They are often unknown and discovered at the stage of infectious complications. They present big therapeutic challenges especially in the late forms. This observation illustrates a rare case of two post-traumatic para-spinal foreign bodies (fabric, plastic) and a postoperative foreign body (gauze or textiloma) discovered during surgery of a traumatic thoracic spine.

\section{Case Report}

A 32-year-old man, admitted at the third hour for a polytrauma after a road traffic accident. It is a Motorcyclist, which collided with an animal-drawn cart. A metal arm of the cart pierced through the left side of his chest during the accidental collision.

The admission examination noted a penetrating chest wound with dyspnea, a closed trauma of the thoracic spine with spinal cord injury syndrome. ASIA score was A with flaccid paraplegia and anesthesia going up to T10. The gibbosity of the thoracic spine was observed at the level of the fifth and sixth thoracic vertebrae.

The CT scan revealed multiple fractures of ribs associated with compressive left pleural effusion and pulmonary contusion, and an unstable fracture of the fifth (T5) and sixth (T6) thoracic vertebrae (Figure 1) associated with posterior epidural hematoma responsible for bone marrow compression. There was a rounded isodense para-spinal image dotted with small areas of low density, air bubbles, without contrast enhancement.

We lifted the vital emergency by draining the left pleural effusion, debridement of the penetrating chest wound, and administering Broad-spectrum antibiotic therapy (Ceftriaxone and Metronidazole).

Fourteen days after this initial management, the general condition of the patient was good. He was afebrile. The post traumatic thoracic wound and the thoracotomy had healed well. A painful gibbosity of the spine was observed at the level of the fifth and sixth thoracic vertebrae. The neurological deficit remained stable (ASIA A). Biological analysis (leukocytes, blood sedimentation rates and C-reactive protein levels) had normal values. Control Chest X-ray showed the regression of the left pleural effusion. We decided to stabilize the spine by bone synthesis. After a posterior approach of the thoracic spine centered 

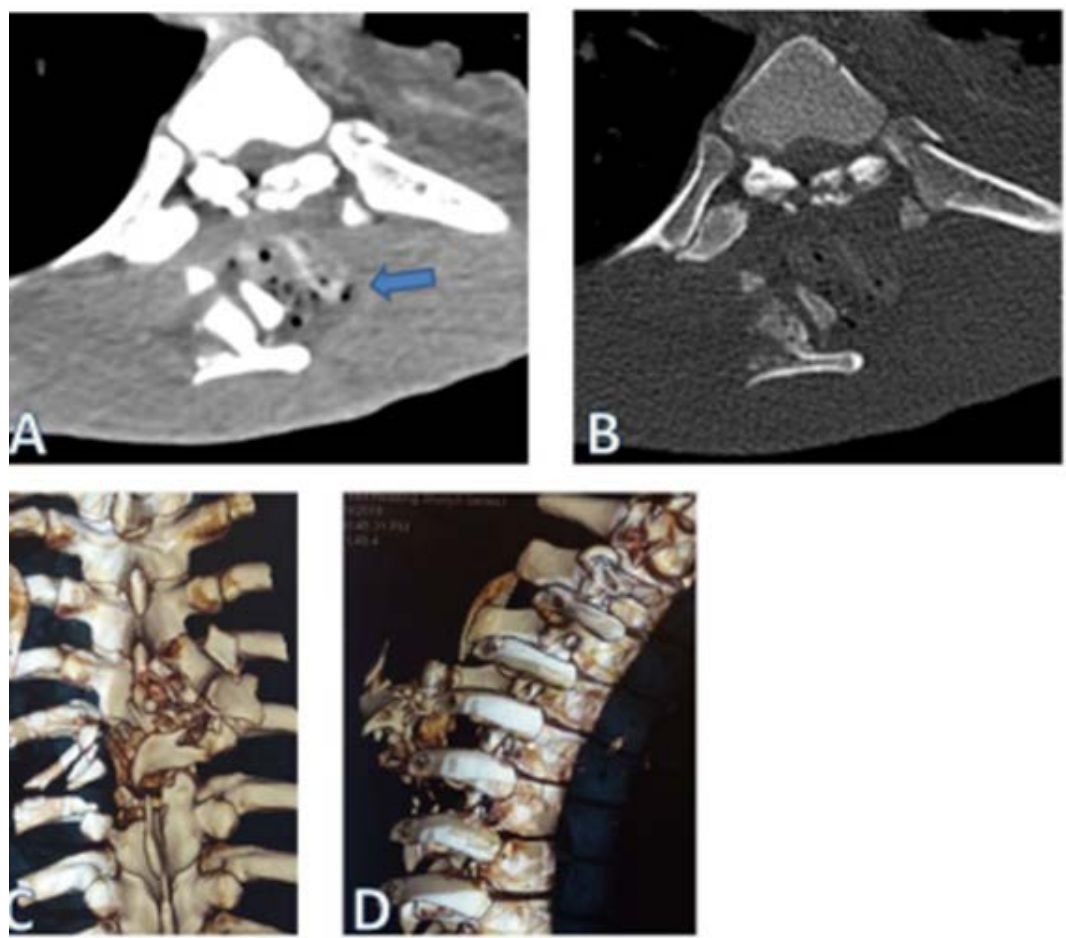

Figure 1. Axial view showing a rounded image dotted with areas of low density, air bubbles (A). Intracanal multiple bony fragments (B), Unstable fracture of the 5th and 6th thoracic vertebrae on a $3 \mathrm{D}$ reconstruction posterior view of thoracic spine associated with ribs fractures $(\mathrm{C})$. Broken crushed fragment of the spine behind which a foreign body could be evoked on a profile view (D).

on the fifth and the sixth thoracic vertebra, we discovered three para-vertebral foreign bodies. They were a piece of plastic (Figure 2(A) and Figure 2(B1)) that appeared after muscular disinsertion, a piece of fabric (Figure 2(B2)) and finally a gauze probably left in place after the debridement of the thoracic wound (Figure 2(B3)). There was a $3 \mathrm{~cm}$ long hole on the left para-vertebral side communicating with the thoracic cavity (Figure $2(\mathrm{C})$ ) from which nauseating frank yellow pus flowed. Removal of a crushed fragment consisting on a T5 right slide and two T6 slides allowed evacuation of chronic intra-spinal extradural hematoma. Given the infectious context, no implant had been put in place. We had opted for a thoraco-lumbar rigid orthosis to stabilize the spine. A chest tube has been put in place. Broad-spectrum antibiotic therapy was continued and later on adapted to the antibiogram result. Enterobacter spp. was isolated and susceptible to imipenem. The immediate post operative follow-ups were simple. The patient was then referred to thoracic surgery. The resolution of the pain was observed ten days after the removal of the foreign bodies. The neurological state remained stationary (ASIA A).

\section{Discussion}

The incidence of foreign bodies ranges from $1 / 1000$ to $1 / 10,000$, of which $80 \%$ are textiloma [1] [4] [5]. Foreign bodies were frequently connected to abdominal 


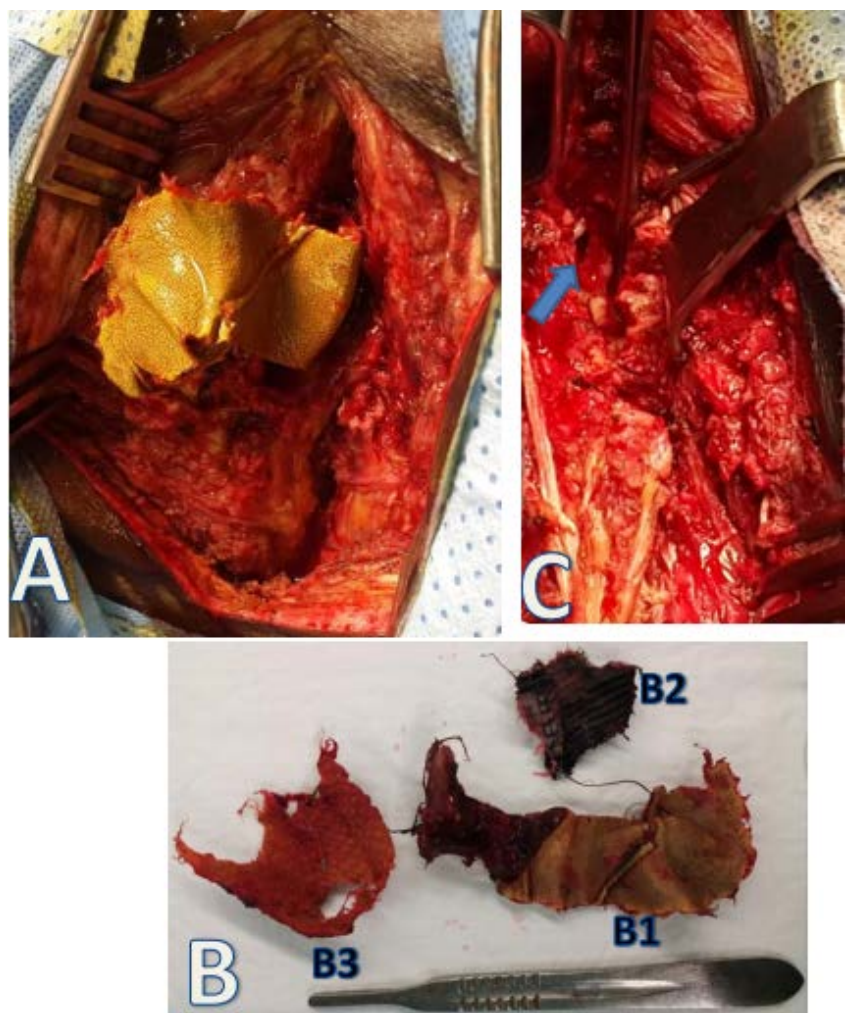

Figure 2. Foreign bodies extracted during surgery. Piece of plastic appearing after a posterior approach (A and B1), a piece of fabric (B2) and finally a gauze (B3).

or thoracic surgery [5] [6] [7] rarely after surgery on the spine [2] [3]. Most para-spinal foreign bodies are poorly documented [8]. Although commonly encountered in daily practice a low case rate is reported in literature [8], about $6 \%$ for spinal localizations [1] [8]. Our case illustrates a rare presentation of para-spinal foreign bodies, following a penetrating thoracic trauma associated with a fabric [gauze] left in place after a first emergency surgery. These strange bodies were accidentally discovered during spine surgery. Several reasons have been reported as possible causes of intraspinal foreign bodies, including surgical, patient and human factors [4] [6]. They are more common after emergency surgery or an unexpected change in the surgical procedure such as hemorrhage [8], rarely post-traumatic. The two foreign bodies went unnoticed after an incomplete exploration of the penetrating wound of the thorax during debridement. Gauze used for hemostatic purpose had been forgotten in the deep hemorrhagic wound. The stamping procedure would have contributed to pushing back these foreign bodies deeply. They probably would have migrated and continued their course to the thoracic spine through the posterior hole caused by the trauma. The dorsal para-spinal migration described in this article could be evoked as a mechanism, although exceptional. Gauze used is generally useful to stop hemorrhage [6] [7]. Although precautions are taken to avoid leaving such materials, errors occur. These forgotten materials can lead to local infection by reaction of the surrounding tissue, with an exudative reaction and formation of ab- 
scess [7] [8]. The last one would be the most frequent and the most dangerous. In reaction to fibrous aseptic tissue, the formation of a fibrous capsule would lead to granuloma [1] [5] [7]. In this case, the patient may remain asymptomatic for days to years [4] [8]. Sometimes the foreign body can be accidentally discovered during a routine radiological examination [9]. The circumstances of discovery are often the appearance of a persistent infectious syndrome [10] preceded by persistent pain [1] [7]. This persistent characteristic pain described in the literature [3] [11] seemed more related to spinal trauma in our case. No clinical or biological infection syndrome was observed. This can be observed in some cases [12]. The absence of fever could be related to antibiotic therapy instituted upon admission and continued for ten days. The empiric administration of antibiotic for any temperature rise in the postoperative period or after open trauma is often the cause of delayed diagnosis [1] [12]. The reasons for this delay in diagnosis include the low index of suspicion and the time interval before the onset of symptoms [9].

Preoperative diagnosis is possible, but very difficult by imaging. There are no specific signs [4] [7] [8] [13]. Foreign bodies made of cotton have fibers which characteristic features can appear on standard radiography [13]. In this case, the standard radiography did not allow evoking the presence of foreign bodies. Standard control radiography showed complete regression of pleural effusion after thoracic drainage. Thoracic computed tomography (CT) is considered to be the best in detection of textilomas and their possible complications [4] [12]. It shows the character, heterogeneous, high-density, and lack of contrast enhancement [4] [7] [13]. For our patient, it showed a para-spinal image with heterogeneous iso-density characters and dotted with low-density small air bubbles without contrast enhanced. This image made it possible to suspect the presence of a foreign body. The introduction of MRI, which seems more efficient, has made it possible to diagnose most foreign bodies [7] [13]. It allows an excellent visualization of the characteristic internal structure of the foreign body in three planes of space. Generally, most lesions caused by foreign body [textiloma] are hypointense on T1-weighted images and hyperintense on T2-weighted images [11] [13]. Para-vertebral mass may have an intermediate signal intensity that would be due to the in situ duration of foreign bodies [11] [12]. It can be raised strongly on the periphery in injected T1-weighted [12]. The appearance of an inflammatory reaction beyond 15 days could be an explanation. Imaging features are variable and nonspecific; thus the differential diagnosis can be made with a solid tumor or an epidural abscess [4] [6] [8] [13]. Similarly, in patients with a history of surgery or open trauma, the presence of foreign bodies should be discussed and considered in the diagnostic strategy [8] [9] [12]. The physical examination, laboratory results and imaging may lead to the surgical indication [1] [7]. However, in our case, the patient had no infectious syndrome or suggestive image on imaging. It was an intraoperative discovery. Confirmation is provided by surgical exploration [6] [10]. The identification of a germ had allowed us to 
adapt the antibiotherapy in our patient. Administration of broad-spectrum antibiotics initially used in routine practice will then be replaced according to the results of biology and susceptibility test [2] [7] [10]. Appropriate antibiotic therapy is recommended when a suppurative complication is present or suspected [2] [3] [8]. However, the germ is not always identified [2] [8] [9]. In our case the identified germ is part of the germs usually reported in published journals [1] [7] [8]. These include Staphylococcus aureus, Klebsiella oxytoca, Enterobacter spp., Streptococcus, Enterobacter aerogenes [2] [7] [8] [9].

The treatment of foreign bodies can only be surgical [10]. The difficulty of their extraction depends essentially on the delay between the date of their introduction and their discovery [4] [11] [12]. Thus, foreign bodies discovered before the 15th postoperative day are easily treated because the inflammatory and infectious mechanisms are not yet installed. In our case, the surgery was performed before this time. Extraction of foreign bodies was easy and local complication at the beginning stage. Compared to other series where the discovery was late, surgery difficult [8] [12]. The encapsulation process and infection led to fibrosis, sclerosis, and even tissue destruction, leading to a hemorrhagic and extensive surgery [8] [12]. Early surgery is recommended in the management of spinal cord injury. The rarity of implants in our practice justifies a long delay before surgery.

\section{Conclusion}

Traumatic or postoperative foreign bodies are much more common than they are reported. Imaging sometimes makes it possible to suspect the non-metallic foreign body. The diagnosis of these non-metallic foreign bodies is almost always an intraoperative surprise. These foreign bodies are sources of infection making their management difficult. Their best treatment is preventive. Careful exploration of penetrating wounds, labeling and counting of gauze used intraoperatively, and careful inspection of the surgical field before closure are still important basic rules in surgery. Complications caused by foreign bodies are source of mortality and serious medico-legal implications.

\section{Informed Consent}

Written Informed consent has been obtained from the patient for publication of this manuscript and all accompanying images.

\section{Conflicts of Interest}

The authors have no conflicts of interest to declare.

\section{References}

[1] Okten, A.I. and Gezercan, M.Y. (2006) Textiloma: A Case of Foreign Body Mimicking a Spinal Mass. European Spine Journal, 15, S626-S629.

https://doi.org/10.1007/s00586-006-0136-6 
[2] Patel, A.R., Alton, T.B., Bransford, R.J., Lee, M.J., Bellabarba, C.B. and Chapman, J.R. (2014) Spinal Epidural Abscesses: Risk Factors, Medical versus Surgical Management, a Retrospective Review of 128 Cases. Spine Journal, 14, 326-330. https://doi.org/10.1016/j.spinee.2013.10.046

[3] Johnson, K.G. (2013) Spinal Epidural Abscess. Critical Care Nursing Clinics of North America, 25, 389-397. https://doi.org/10.1016/j.ccell.2013.04.002

[4] Kim, H.S., Chung, T.S., Suh, S.H. and Kim, S.Y. (2007) MR Imaging Finding of Paravertebral Gossypiboma. American Journal of Neuroradiology, 28, 709-713.

[5] Karabulut, N., Duygu, H. and Kıroğlu, Y. (2011) CT Features of Intrathoracic Gossypiboma (Textiloma). Diagnostic and Interventional Radiology, 17, 122-124.

[6] Naama, O., Quamous, O., Elasri, C.A., Boulahroud, O., Belfkih, H., Akhaddar, A., et al. (2010) Textiloma: An Uncommon Complication of Posterior Lumbar Surgery. Journal of Neuroradiology, 37, 131-134. https://doi.org/10.1016/j.neurad.2009.04.003

[7] Chen, J.M., Wang, Z.Y. and Ni, G.X. (2016) Thoracic Spinal Epidural Abscess Caused by Fishbone Perforation. A Case Report and Review of Literature. Medicine, 95, e5283. https://doi.org/10.1097/md.0000000000005283

[8] Şahin, S., Atabey, C., Şimşek, M. and Naderi, S. (2013) Spinal Textiloma (Gossypiboma): A Report of Three Cases Misdiagnosed as Tumour. Balkan Medical Journal, 30, 422-428. https://doi.org/10.5152/balkanmedj.2013.8732

[9] Akhaddar, A., Boulahroud, O., Naama, O., Al-bouzidi, A. and Boucetta, M. (2012) Paraspinal Textiloma after Posterior Lumbar Surgery. A Wolf in Sheep's Clothing. World Neurosurgery, 77, 375-380. https://doi.org/10.1016/j.wneu.2011.07.017

[10] Ziad, T., Rochdi, Y., Benhoummad, O., Nouri, H., Aderdour, L. and Raji, A. (2014) Retropharyngeal Abscess Revealing a Migrant Foreign Body Complicated by Mediastinitis: A Case Report. Pan African Medical Journal, 19, 125. https://doi.org/10.11604/pamj.2014.19.125.5334

[11] Atabey, C., Turgut, M. and Ilica, A.T. (2009) Retained Surgical Sponge in Differential Diagnosis of Paraspinal Soft-Tissue Mass after Posterior Spinal Surgery: Report of Eight Cases. Neurology India, 57, 320-323. https://doi.org/10.4103/0028-3886.53289

[12] Kucukyuruk, B., Biceroglu, H., Abuzayed, B., Ulu, M.O. and Kafadar, A.M. (2010) Paraspinal Gossybipoma: A Case Report and Review of the Literature. Journal of Neurosciences in Rural Practice, 1, 102-104. https://doi.org/10.4103/0976-3147.71725

[13] Hakan, T., Aydoseli, A., Demir, K. and Aker, F. (2009) Clinical, Pathological and Radiological Features of Paraspinal Textiloma: Report of Two Cases and Review of the Literature. Neurologia i Neurochirurgia Polska, 43, 475-478. 